Cahiers $d u$ MONDE RUSSE

\section{Cahiers du monde russe}

Russie - Empire russe - Union soviétique et États indépendants

45/3-4 | 2004

Varia

\title{
E. Ju. Zubkova, L. P. Košeleva, G. A. Kuznecova, A. I. Minjuk, L. A. Rogovaja, eds., Sovetskaja Žizn', 1945-1953
}

\section{Bella Ostromoukhov}

\section{(2) OpenEdition}

\section{Édition électronique}

URL : https://journals.openedition.org/monderusse/4206

DOI : 10.4000/monderusse.4206

ISSN : $1777-5388$

Éditeur

Éditions de l'EHESS

\section{Édition imprimée}

Date de publication : 1 juillet 2004

Pagination : 710-712

ISBN : 2-7132-2009-2

ISSN : $1252-6576$

\section{Référence électronique}

Bella Ostromoukhov, «E. Ju. Zubkova, L. P. Košeleva, G. A. Kuznecova, A. I. Minjuk, L. A. Rogovaja, eds., Sovetskaja žizn', 1945-1953», Cahiers du monde russe [En ligne], 45/3-4 | 2004, mis en ligne le 03 juin 2009, consulté le 03 septembre 2022. URL : http://journals.openedition.org/monderusse/4206 ; DOI : https://doi.org/10.4000/monderusse.4206

Ce document a été généré automatiquement le 3 septembre 2022.

Tous droits réservés 


\title{
E. Ju. Zubkova, L. P. Košeleva, G. A. Kuznecova, A. I. Minjuk, L. A. Rogovaja, eds., Sovetskaja Žizn', 1945-1953
}

\author{
Bella Ostromoukhov
}

\section{RÉFÉRENCE}

E. Ju. ZUBKOVA, L. P. KOŠELEVA, G. A. KUZNECOVA, A. I. MINJUK, L. A. ROGOVAJA, eds., Sovetskaja Žizn', 1945-1953 (La vie soviétique, 1945-1953). Moscou, ROSSPEN, 2003, 719 p. (Serija « Dokumenty sovetskoj istorii »)

1 C'est « le quotidien soviétique, la vie de tous les jours des gens ordinaires » (p. 5), qui fait l'objet de ce recueil de documents. Objet familier pour Elena Zubkova, qui lui a déjà consacré plusieurs études ${ }^{1}$, mais objet nouveau, d'après les auteurs, pour un recueil de ce type. En effet, refléter le quotidien d'une époque à travers des documents d'archives est une tâche délicate qui peut se heurter à un certain nombre de problèmes épistémologiques.

2 Premièrement, un tel projet suppose une visée globale: présenter la "vie soviétique » signifie plonger le lecteur dans toute la diversité des « quotidiens » de l'après-guerre. Les auteurs du recueil tentent d'y parvenir en multipliant et en croisant les sources. Sélectionnés dans les fonds du RGASPI (fonds du CC du PCUS et du Komsomol), du GARF (fonds du Soviet suprême, du Conseil des ministres, des ministères de la Justice, de l'Intérieur et du Conseil pour les affaires de l'Église orthodoxe) et du RGAE (fonds du Gosplan, du ministère des Finances et du Bureau central de la statistique), les documents sont de nature diverse. Des lettres de particuliers adressées aux instances officielles et des extraits de correspondances privées interceptées par la censure sont confrontés aux rapports et comptes rendus provenant des organes de base du parti, des organes de l'État, 
du Komsomol et des syndicats. À travers ces textes, officiels ou non, ayant des visées différentes, on peut percevoir à la fois une diversité d'approches du quotidien et une convergence de thématiques. Des tableaux statistiques secrets, destinés à l'usage interne et donc sans doute plus fiables que les données rendues publiques, viennent contextualiser les propos tenus par de simples citoyens. Le quotidien apparait donc comme une somme de regards subjectifs replacés dans un contexte global.

Une deuxième difficulté inhérente à ce type de recueil est celle de sa structure. En effet, comment dégager des domaines distincts dans ce qui, par définition, est un entrelacs de questions intimement liées entre elles? Les auteurs choisissent de dégager quelques " sphères ", tout en prévenant le lecteur, dès l'introduction, du caractère conventionnel d'une telle division. En effet, la répartition des documents en huit parties - « Le quotidien dans l'après-guerre ", "Stratégies de survie ", "L'état d'esprit des différents groupes de population", "La place de l'homme au sein du système soviétique", "Société et économie », "Guerre et paix ", "Religiosité populaire et Église orthodoxe russe », "Vie privée » - n'est que partiellement justifiée.

Les documents de la première partie présentent, en guise d'introduction, un faisceau de thèmes qui seront développés dans le reste du recueil. Il s'agit notamment de problèmes liés aux enfants, tels que la dégradation du niveau de l'enseignement scolaire ou l'accroissement du nombre d'enfants abandonnés, potentiellement délinquants; de plaintes de citoyens concernant les pots-de-vin exigés par de hauts responsables de l'industrie ou des représentants de l'État ; de l'enrichissement démesuré des dirigeants et autres injustices sociales. On y expose par ailleurs les variations de l'état d'esprit de la population dans le domaine politique, vues au travers des questions posées lors de débats organisés par le parti ou glanées par les informateurs sur les lieux de travail.

Le titre de la deuxième partie, "Stratégies de survie », est prometteur : on s'attend à voir transparaitre, à travers les documents, les ruses utilisées par la population afin de déjouer les difficultés matérielles. Malheureusement, ce sont surtout ces dernières qui sont exposées. Un chapitre est consacré aux problèmes liés à la nourriture et à l'habillement, un autre porte sur les défaillances de l'approvisionnement et la famine de 1946-1947, un troisième contient des données statistiques sur l'habitat (offre et état de salubrité), d'autres encore font état d'une recrudescence de la criminalité dans certaines villes. Tous ces documents font apparaitre une multitude de questions : comment nourrir ses enfants avec des cartes de rationnement qui prévoient une quantité de nourriture insuffisante? comment travailler lorsqu'on est affaibli par la faim ? comment participer à la vie sociale si on n'a pas les moyens d'acheter des vêtements? où prendre l'argent pour les emprunts d'État? comment garder sa dignité d'homme soviétique s'il n'y a pas de bains publics dans la ville? que faire lorsqu'on n'ose pas quitter son appartement de peur d'être cambriolé ? Les lettres ne proposent pas d'autre solution que le suicide ou un détournement de la loi - « on n'a plus qu'à mourir ou à voler », « je n'ai plus qu'à me pendre »-, ce qui offre peu de pistes sur les stratégies de survie à proprement parler. Ces textes présentent donc plutôt un point de départ éventuel pour une nouvelle réflexion dont les sources pourraient relever, par exemple, de l'histoire orale.

Dans la partie suivante, ces difficultés matérielles, ces tensions sociales et la réaction de la population face à ces conflits sont déclinées en fonction de certaines catégories sociales: paysans, ouvriers et population urbaine, soldats revenus du front, jeunes et " citoyens aux droits restreints ". Deux thèmes récurrents feront l'objet des deux parties suivantes: d'une part, la place dévolue à l'individu dans un système où le sacrifice 
personnel est exigé sans contrepartie de l'État, et, d'autre part, la situation économique qui ne répond pas aux efforts fournis. Le premier est central dans la quatrième partie, consacrée au statut de l'« opinion publique»: le «peuple vainqueur» veut avoir son mot à dire dans certaines questions politiques, comme les élections au Soviet suprême de 1946 ou la convocation du XIXe Congrès du PCUS en 1952. Un chapitre très intéressant, consacré aux réactions de la population face à la justice, montre l'étonnement des kolkhoziens devant des procès et des condamnations perçus comme infâmes ; un autre, non moins passionnant, laisse entrevoir l'indignation que suscitent les discriminations opérées contre les Juifs et les " peuples punis ».

7 La partie suivante montre l'incidence de la situation économique de la population sur cette «opinion publique». Une série de tableaux du Bureau central de la statistique retraçant l'évolution du budget des particuliers et de l'État pendant cette période précède des documents montrant les réactions du public à la baisse des prix, aux emprunts d'État et à la réforme monétaire de 1947. On voit un certain nombre d'initiatives naître au sein de la population, projets de réformes économiques parfois fantaisistes, mais qui constituent une tentative de dialogue avec les autorités. Les dysfonctionnements économiques sont souvent mis sur le compte de l'ennemi extérieur, thème que l'on retrouve dans la partie suivante, consacrée à la rumeur publique concernant la guerre froide et le conflit en Corée.

8 Les deux dernières sections portent sur l'action de l'État dans la sphère de l'intime. Dans le domaine religieux, des rapports issus de sources diverses montrent à la fois une recrudescence de la foi dans certaines régions et le dispositif de surveillance mis en place pour l'endiguer. La dernière partie, qui traite de la vie familiale, évoque les tentatives des citoyens pour contourner des interdits comme l'avortement ou le mariage avec les étrangers.

9 On voit donc que les différents ensembles qui composent ce recueil constituent, plus qu'une structure rigide, une somme de thèmes qui se recoupent, ce qui entraîne des redondances. Cette forme manque parfois de rigueur, mais elle permet, en revanche, de rendre la complexité du « quotidien ».

10 C'est dans la définition de celui-ci que réside la difficulté majeure de ce type d'ouvrage. En effet, qu'est-ce qui constitue la vie de tous les jours? D'après certains historiens russes contemporains ${ }^{2}$, il s'agit des objets qui entourent l'individu, de l'organisation de l'espace domestique, de l'environnement de travail et des loisirs. Cette conception ne recoupe qu'en partie celle du présent recueil : les objets concrets y apparaissent le plus souvent en creux, lorsqu'il s'agit d'un manque, comme la pénurie de vêtements ou de logements convenables. Les documents rassemblés ne permettent de se représenter la matérialité du quotidien que dans des situations de crise. Quant à l'organisation des loisirs ou aux choix culturels de la population, ils sont absents. En revanche, les documents ouvrent sur une autre dimension de la vie de tous les jours, celle de la rumeur populaire, ce qui permet d'appréhender les individus comme des êtres réactifs dotés d'une opinion propre.

11 Une telle approche de la société par le biais de documents mettant en évidence ses carences et ses tensions en induit nécessairement une vision particulière. En effet, certaines catégories se trouvent de fait écartées : ainsi les enfants ne sont mentionnés que lorsqu'ils représentent un danger aux yeux de l'ordre public ou une charge matérielle insupportable pour leurs parents; on ne sait presque rien du quotidien des démobilisés lorsqu'ils ne sont pas invalides, ni de la population citadine autre 
qu'ouvrière... Les auteurs du recueil sont conscients de cette déformation qu'ils expliquent, dans l'introduction, par la nature même du matériel utilisé : «Si un homme prenait sa plume, c'était surtout pour parler de ses problèmes». Cependant, cet ouvrage ne prétend pas à l'exhaustivité. Réunissant un ensemble immense de témoignages, il propose au lecteur une multitude de questions à développer à partir de nouvelles sources.

\section{NOTES}

1. Elena Zubkova, Obščestvo i reformy, 1945-1964 (La société et les réformes, 1945-1964), Moscou, Rossija molodaja, 1993, et surtout Poslevoennoe obščestvo : politika i povsednevnost', 1945-1953 (La société d'après guerre : la politique et le quotidien, 1945-1953), Moscou, ROSSPEN, 2000.

2. Voir par exemple N. B. Lebina, A. N. Čistikov, Obyvatel' i reformy, kartiny povsednevnoj žizni gorožan $v$ gody nepa $i$ hruščevskogo desjatiletija (L'homme ordinaire face aux réformes, tableaux de la vie quotidienne des citadins durant la NEP et la décennie khrouchtchévienne), Saint-Pétersbourg, "Dmitrij Bulanin », 2003. 